\title{
Theory of Direct Interband Transition due to Electron-Phonon Interactions in Two Weak-Coupling Schemes.
}

Saxg Don Chor and OK Hee Chujg

Department of Physics, Kyungpook National Lniversity - Taegu, South Korea

(Lett. Nuovo Cimento, 38, 221 (1983))

PACS. 63.20. .. Phonons and vibrations in crystal lattices.

In eq. (16) (pag. 223) the summation sign $\sum_{\alpha}$ must be omitted.

(C) by Sooleta Italiana di Fisica 